\title{
Finite element modelling of the resistive heating of disposable molecular diagnostics devices
}

\author{
T. Pardy ${ }^{1}$, T. Rang ${ }^{1} \&$ I. Tulp ${ }^{2}$ \\ ${ }^{1}$ Thomas Johann Seebeck Department of Electronics, \\ Tallinn University of Technology, Estonia \\ ${ }^{2}$ SelfD Technologie GmbH, Germany
}

\begin{abstract}
We present a finite element model for the simulation of the resistive heating of microchannels in disposable molecular diagnostics devices by means of various resistive heating elements, and demonstrate the validity of this model through experiments. Polyimide etched foil heaters and heaters based on positive temperature coefficient (PTC) ceramics could be simple, cost-efficient and robust means of heating disposable Lab-on-a-Chip devices that depend on maintaining a given temperature range in the channels for an extended time (i.e. 15-60 minutes) with a precision of $\pm 1-5^{\circ} \mathrm{C}$. However, the design of these devices is a slow and costly process due to the many design factors involved. We demonstrate a finite element model that could reduce design time and save on prototyping costs. The validity of the model is demonstrated through various PMMA test structures designed to emulate a Lab-on-a-Chip device capable of supporting isothermal nucleic acid amplification reactions. With our experimental setups we were able to produce and maintain target temperatures for over 45 minutes with a precision of at most $\pm 1^{\circ} \mathrm{C}$ deviation from the set point in a battery-operated test system without the use of a thermostat. The physical parameters of the available resistive heating elements were used in our finite element model, and the results of the simulations compared to experimental data.

Keywords: lab-on-a-chip, resistive heating, microfluidics, computer aided design, finite element modelling.
\end{abstract}




\section{Introduction}

In molecular diagnostics, temperature control is required for a wide variety of assays, particularly immunoassays and nucleic acid amplification assays. These assays require complex liquid handling, usually done via microfluidics. For a portable, disposable device, it is necessary to have a heating solution with low power dissipation, minimal space requirements and low cost per device. These criteria are easily satisfied by electrical heaters, however, including control circuitry increases development costs and complexity, while having a negative effect on the robustness of the device. For a point-of-care test intended for use at home, minimal complexity is essential to rule out possible errors. To avoid the need for control circuitry, two options are available: self-regulating heating elements, such as PTC (positive temperature coefficient) ceramic pellets or PTC resistance wires, or optimizing the heating solution so that the heat output saturates in a predefined temperature range. The latter solution is possible through geometry optimization, but will limit application of the device to a well-defined ambient temperature range.

However, the topic of integrated, portable (preferably disposable) electrical microheaters for microfluidics has not been researched extensively according to the available literature. The group of Wyzkiewicz et al. [1] proposed a selfregulating heating solution for microfluidics that utilises PTC resistance wires. One of their demonstration setups used printed circuit boards (PCB) as substrate, consisting of interdigitated tracks of carbon-copper-silver, which could be a reasonably cheap microheating solution for disposable molecular diagnostics. The demonstrated setup had an output temperature range of $30-70^{\circ} \mathrm{C}$, suitable for a wide variety of assays.

However, looking at microheating solutions from an industrial perspective, developing a custom-tailored heating solution may be less cost-efficient than choosing a commercially available one and adapting the device geometry and parameters accordingly. In any case, several prototyping stages and device optimization are necessary, and the higher the number of steps, the more costly development is. To reduce these costs, computer aided prototype validation may be employed, using finite element models to simulate and predict device behaviour for a particular design. This approach, coupled with computer aided design may significantly save on time and human labour during development.

The goal of the work described in this paper was to propose an option for the finite element modelling of microheating in disposable molecular diagnostics devices using various commercially available resistive heating elements. The proposed model was tested for multiple experimental setups, emulating microfluidics-based molecular diagnostics devices, using two different heating elements. Additionally, the possibility of battery operation for full portability was evaluated. Furthermore, the experimental setups demonstrated were intended to maintain the target temperature within $\pm 1^{\circ} \mathrm{C}$ around a set point without the use of external temperature control. 


\section{Theoretical background}

\subsection{Self-regulating heating elements}

In one of the experimental setups discussed in this paper, resistive elements were based on positive thermal coefficient (PTC) ceramics, which self-regulate their temperature output. This regulation in effect occurs via a rapid increase in resistance with temperature, which essentially regulates the current passing through the heating element. Although most of the manufacturers of currently commercially available heaters do not disclose material data, the most typically used material is doped (3- or 5-valent dopants) polycrystalline barium titanate $\left(\mathrm{BaTiO}_{3}\right)$ ceramic, mentioned in detail by Shioi et al. [2].

The onset of the PTCR effect is at the Curie temperature $\left(T_{c}\right)$ of the material, the transition temperature from ferroelectric to paraelectric phase. This temperature can be adjusted by selecting the proper dopant, such as strontium or lead as detailed by Huybrechts et al. [3], in turn determining the onset of the PTCR effect, and thus, the set point (or temperature range) of the heating element.

In order to characterise PTC heaters and the PTCR effect in practice, only the temperature coefficient of resistance $\alpha[1 / \mathrm{K}]$ and the initial resistance $R_{0}[\Omega]$ are needed, but the latter is measured during recording the data points necessary for calculating the temperature coefficient, where $\alpha$ may be perceived as the slope of the linear curve with which the resistance of the PTC material is approximated.

\subsection{Finite element modelling}

The basis for modelling resistive heating is provided by Joule's first law, which states that electric currents passing through a conductor generate heat, which is proportional to the resistance of the conductor $R[\Omega]$ and the current $I[\mathrm{~A}]$ passing through it (e.g. Meier [4]):

$$
Q \propto I^{2} \cdot R
$$

$Q[\mathrm{~W}]$ is the energy (heat) released, and if direct current is applied, can be expressed via the formula of power: $P=V \cdot I=I^{2} \cdot R$. The initial assumption is made that liquid in the microchannel is either slow enough to be considered stationary, or is kept stationary for the duration of heating.

Therefore the model consists of two equations: heat generation is expressed by the formula of power (eqn. (2)), and then heat is propagated through the geometry of the model by the heat transfer equation, eqn. (5). Space-dependence in the model is defined by a Cartesian coordinate system with 3 axes $x[\mathrm{~m}], y[\mathrm{~m}], z[\mathrm{~m}]$, whereas time-dependence is described by time $(t)$ in seconds. Scaling is permitted. Heat generation is expressed via the aforementioned formula of power, written in a differential form for the volume of the heater (Meier [4]):

$$
\frac{d P}{d V}=J \cdot E
$$

where $P[\mathrm{~W}]$ is the power, $\mathrm{V}\left[\mathrm{m}^{3}\right]$ the volume of the heating element, $\mathrm{J}\left[\mathrm{A} / \mathrm{m}^{2}\right]$ the current density, $E[\mathrm{~V} / \mathrm{m}]$ the electric field. Current densities and the electric 
field used for calculating heat generation are in turn calculated from the following set of equations (Griffiths [5]):

$$
\begin{gathered}
\nabla J=Q_{j}, \\
J=\sigma E+\frac{\partial D}{\partial t}+J_{e}, \\
E=-\nabla V .
\end{gathered}
$$

where $\mathrm{J}\left[\mathrm{A} / \mathrm{m}^{2}\right]$ is the current density, $Q_{j}\left[\mathrm{~A} / \mathrm{m}^{3}\right]$ are current sources (sinks), E the electric field, $\mathrm{D}$ the electric displacement field $\left[C / \mathrm{m}^{2}\right], J_{e}\left[\mathrm{~A} / \mathrm{m}^{2}\right]$ an externally generated current density (if there is one), $V[\mathrm{~V}]$ the potential drop in every direction, $\sigma[\mathrm{S} / \mathrm{m}]$ the conductivity of the material at the point of the geometry for which the equations are solved. Materials in the simulated geometry are therefore characterized electrically through their conductivity. The above set of equations (eqn. (3)) relies on the continuity equation related to charge conservation (Griffiths [5]):

$$
\nabla J=-\frac{\partial \rho}{\partial t}
$$

The above continuity equation states that charge density $\rho\left[\mathrm{C} / \mathrm{m}^{3}\right]$ at a point only changes if current flows into or out of the point.

The heat transfer equation is used in the following form (Kandlikar [6]):

$$
\rho C_{p} \frac{\partial T}{\partial t}-\nabla \cdot(-\mathrm{k} \nabla \mathrm{T})=\mathrm{Q}
$$

where $\rho\left[\mathrm{kg} / \mathrm{m}^{3}\right]$ is the fluid density, $C_{p}[\mathrm{~J} / \mathrm{kg} \mathrm{K}]$ the specific heat capacity at constant pressure, $T[\mathrm{~K}]$ the absolute temperature in the system, $k[\mathrm{~W} /(\mathrm{mK})]$ the thermal conductivity, and $\mathrm{Q}\left[\mathrm{W} / \mathrm{m}^{3}\right]$ is the heat source (sink), which in this case is calculated as the power of the heating element as a whole. With respect to heat transfer, materials of the simulated geometry are simulated through the aforementioned quantities $\left(\rho, C_{p}, k\right)$, solved for each spatial point in the geometry.

In case the temperature dependence of resistivity is taken into account, the wellknown formula for calculating temperature dependent resistivity defines changes $(\operatorname{Rood}[7])$ :

$$
\rho(T)=\rho_{0} \cdot\left[1+\alpha \cdot\left(T-T_{0}\right)\right]
$$

where $\rho_{0}[\Omega \cdot m]$ is the resistivity at ambient (reference) temperature $T_{0}[\mathrm{~K}] . T[\mathrm{~K}]$ is the current temperature of the heating element, calculated from the heat transfer equation, and $\alpha\left[\frac{1}{K}\right]$ is the temperature coefficient of resistivity. Resistivity is derived from resistance in an experimental setup using the following formula (Meier [4]):

$$
\rho=R \frac{A}{l}
$$

where $l(\mathrm{~m})$ is the length (thickness) of the resistive element, and $A\left(\mathrm{~m}^{2}\right)$ its crosssectional area. This formula stands for resistors with uniform cross-sectional area, which is true for all of the devices used in the experimental setups discussed in this paper. 


\section{Experimental setup for model validation}

\subsection{Setup \#1}

For this setup, a commercially available self-regulating resistive heating element was used. The DBK HP04-1/04-24 heater plate consisted of a PTC ceramic heating element, and an aluminium profile with a flat surface on one side. The operating range of this heater was 1-30 $\mathrm{V}_{\mathrm{DC}}$, and its highest measured temperature output was $65.1^{\circ} \mathrm{C}$.

The experimental setup consisted of a PMMA (polymethyl methacrylate) frame designed to house the heating element and the temperature probe adapter, a DC multimeter (Agilent 34410A) and a DC power supply (Agilent E3631A). The plastic temperature probe adapter was designed to imitate the typical size regime of microfluidic chips $(25 \mathrm{~mm} \times 75 \mathrm{~mm}$ ), and contained a $2 \mathrm{~W}$ NTC thermistor with $10 k \Omega$ initial resistance. The adapter was inserted into the slot in the PMMA frame, positioned as close to the flat face of the aluminium profile of the heating element as possible. The assembled test structure is shown in 1(a).
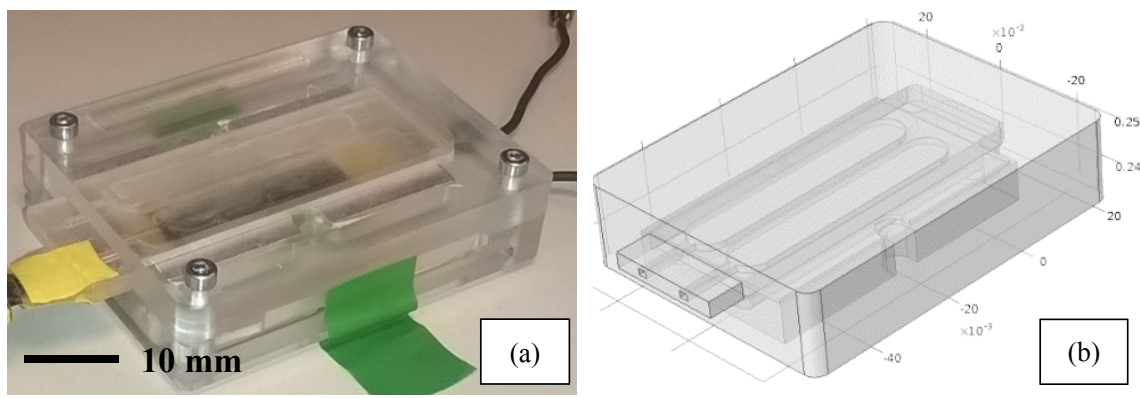

Figure 1: (a) Test structure and (b) model geometry.

\subsection{Setup \#2}

For this setup, a commercially available flexible etched foil heater was used. These resistive heating elements are typically composed of a thin metal film etched to form a heating element, which is sandwiched between layers of electrical insulation. The sample used in the experimental setup was an etched foil heater from Minco Products, Inc. (P/N HR5303R70.2LI2A), in which the etched resistive film was encased in polyimide and silicon rubber sheets. According to manufacturer specifications (Minco [8]), the resistance of this heating element was $70.2 \Omega$.

The experimental setup was similar to Setup \#1, except for the PMMA frame and the heating element. A new, smaller frame was manufactured to house the significantly smaller heating element and the same temperature probe adapter. The assembled experimental setup is shown in Fig. 2(a). 

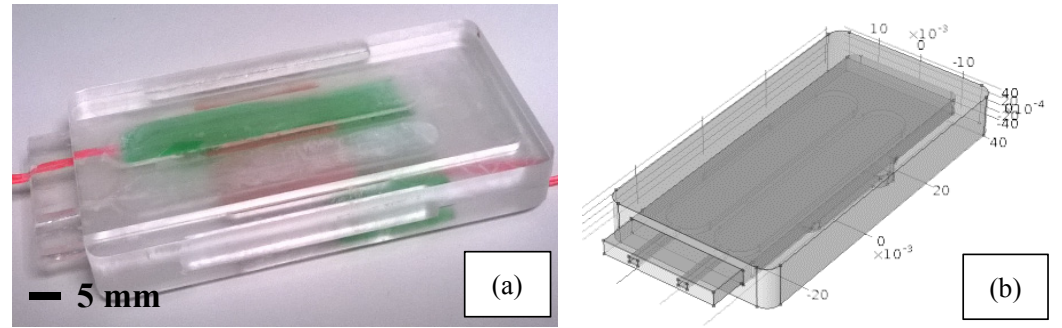

Figure 2: (a) Test structure and (b) model geometry.

\subsection{Setup \#3}

In this setup, the same heating element, temperature probe and PMMA frame were used as in Setup \#1, but instead of a DC power supply, voltage input was provided by a set of $1.5 \mathrm{~V}$ AAA alkaline batteries (LR03-GP-16), which were held in a PMMA battery compartment. The assembled prototype is shown in Fig. 3(a).
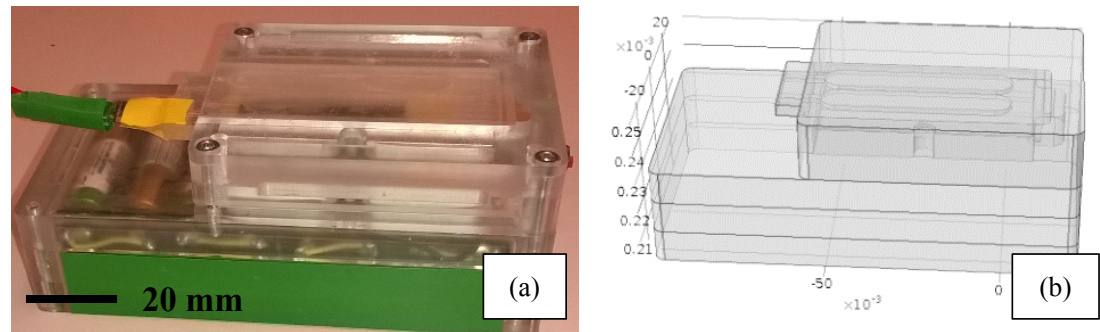

Figure 3: (a) Test structure and (b) model geometry.

This setup was intended as a proof-of-concept experiment for a simple, portable, battery-operated heating solution for microfluidics.

\section{Results and discussion}

\subsection{Electrical characterization of heating elements}

\subsubsection{Setup \#1}

Measurements were conducted using the previously described experimental setup (the power output was provided by the DC power supply), recording the current flowing through the heating element, and calculating resistance using Ohm's law. The temperature coefficient of resistance calculated with eqn. (3) was $0.23 \pm 0.09$ $[1 / \mathrm{K}]$.

The resistance of the PTC ceramic heating element at ambient temperature (considered $20^{\circ} \mathrm{C}$ in this case) was measured and calculated to be $10 \Omega( \pm 0.4 \Omega)$. 


\subsubsection{Setup \#2}

Measurements were conducted in a similar manner as with Setup \#1, recording the current flowing through the heating element. Manufacturer specifications state the resistance of the heating element as $70.2 \Omega$, however, in our experimental setup resistance was measured to be $76.38 \pm 0.37 \Omega$. This deviation from the design value may be due to high manufacturing tolerance levels or conditions specific to our experimental setup. Further investigation may be necessary in the future.

\subsection{Model evaluation}

\subsubsection{Setup \#1}

For the evaluation of model performance, the LAMP (loop-mediated isothermal amplification) reaction, an isothermal nucleic acid amplification method was taken into account. This is a highly sensitive and specific amplification method particularly suitable for use in molecular diagnostics, developed by Eiken Chemical Co. (Nagamine et al. [9]), which requires a $60-65^{\circ} \mathrm{C}$ temperature range to be maintained for 15-60 minutes for a successful amplification of a specific DNA target in a sample.

The required temperature range, $60-65^{\circ} \mathrm{C}$ was taken into account for the evaluation, which was possible to reach experimentally at $24 \mathrm{~V}_{\mathrm{DC}}$ input, so this voltage was used for comparison between model results and measurements. Temperature data was recorded for 45 minutes.

The model was solved using the Joule Heating interface of $\mathrm{COMSOL}^{\circledR}$ Multiphysics Version 4.4. Values for material properties were defined by the software's built-in library. Solution time of this model was 11 minutes 50 seconds ( \pm 16 seconds on average over multiple runs) on a PC with a Core i5-4570 CPU and 16 GB RAM for a single set of input parameters.

Temperature output of the experimental setup saturated around the temperature $64.42 \pm 0.15^{\circ} \mathrm{C}$, which was in the required temperature range, and therefore was defined as the set point of heating Setup \#1. The model estimated temperature output around the set point (reached after $1800 \mathrm{~s}$ in both cases) with an error of $0.31 \pm 0.17^{\circ} \mathrm{C}$ degrees. Comparison of results is shown in Fig. 4 .

Heating in the experimental setup was more rapid than in the model, which was probably attributable to the differences in model geometry. The heating elements could not be disassembled without causing irreversible damage, and the patent of Bohlender and Buchlaub [10] describing the internal structure did not include dimensions, so the geometry of the simulated model was approximate.

\subsubsection{Setup \#2}

The basis for comparison was the same as for Setup \#1, described earlier. The target temperature range, $60-65^{\circ} \mathrm{C}$ was possible to reach at $9 V_{D C}$ input. Since the heat output of the heating element saturated in that temperature regime, external control circuitry was unnecessary to regulate output for the duration of the experiments. Temperature data was recorded for 45 minutes. 


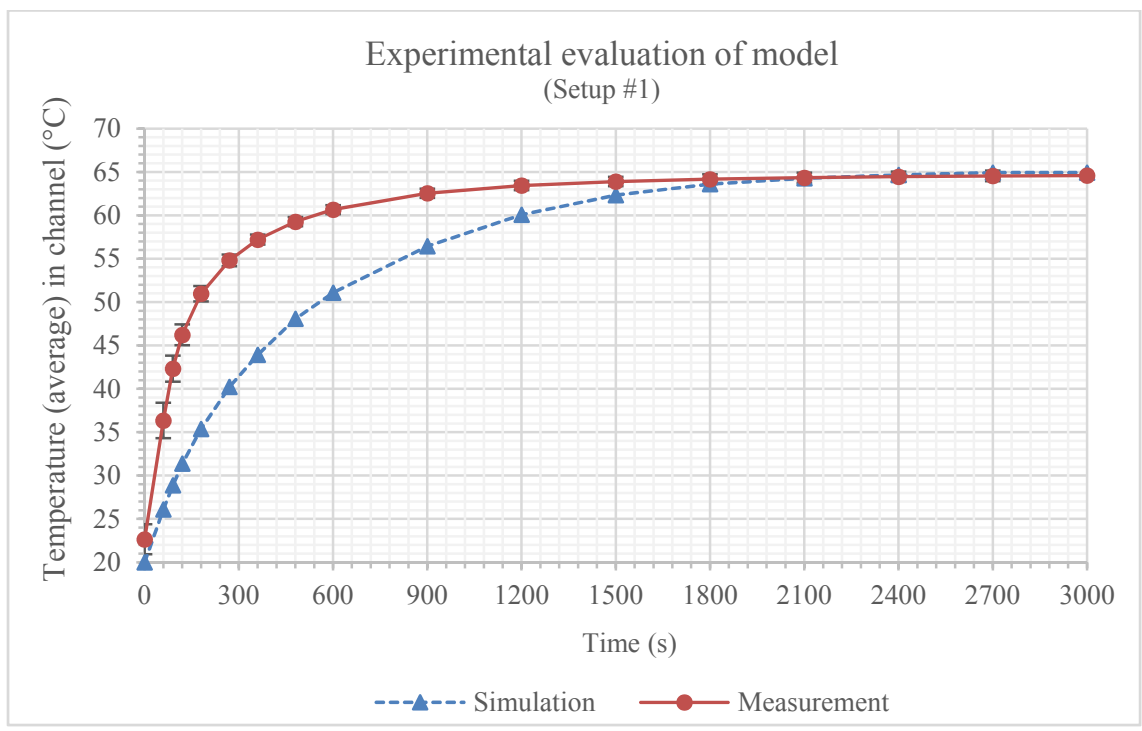

Figure 4: $\quad$ Model evaluation - Setup \#1.

The model was solved using the Joule Heating interface of COMSOL ${ }^{\circledR}$ Multiphysics Version 4.4, using the geometry shown in Fig. 2(b). Values for material properties were defined by the software's built-in library. Solution time of this model was 14 minutes 42 seconds ( \pm 1 minute 31 seconds on average over multiple runs) on a PC with a Core i5-4570 CPU and 16 GB RAM for a single set of input parameters.

The output temperature of the experimental setup saturated around $61.59 \pm$ $0.56^{\circ} \mathrm{C}$, which was in the defined target range, and therefore was accepted as the set point of the heating Setup \#2. The model estimated temperature output with a precision of $1.12 \pm 0.62^{\circ} \mathrm{C}$ around the set point at $1500 \mathrm{~s}$. Although the output temperature kept rising to a perceivable degree, the rate of temperature increase was very low and the highest measured temperature at the 1 hour mark was $63.12^{\circ} \mathrm{C}$, well within the specified $60-65^{\circ} \mathrm{C}$ temperature range.

\subsubsection{Setup \#3}

The basis for comparison was once more the same as for Setup \#1, as well as the hardware and software for solving the model. Solution time was 14 minutes 7 seconds ( \pm 2 minutes 44 seconds on average over multiple runs).

Temperature output of the experimental setup saturated around the temperature $62.74 \pm 0.09^{\circ} \mathrm{C}$, which was in the required temperature range, and therefore was defined as the set point of heating Setup \#3. The model estimated temperature output around the set point (reached after $1800 \mathrm{~s}$ ) with an error of $0.23 \pm 0.11^{\circ} \mathrm{C}$ degrees. Comparison of results is shown in fig. 6 .

The higher estimation error before the set point was due to the same reasons as were explained for Setup \#1. 


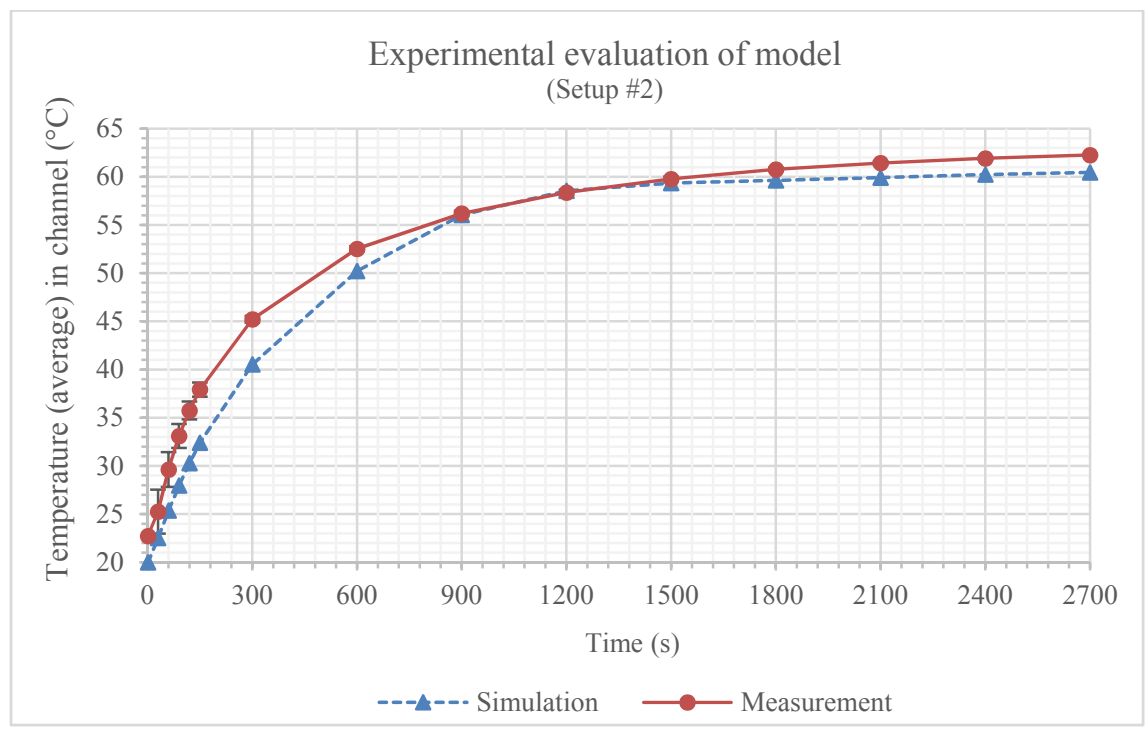

Figure 5: $\quad$ Model evaluation - Setup \#2.

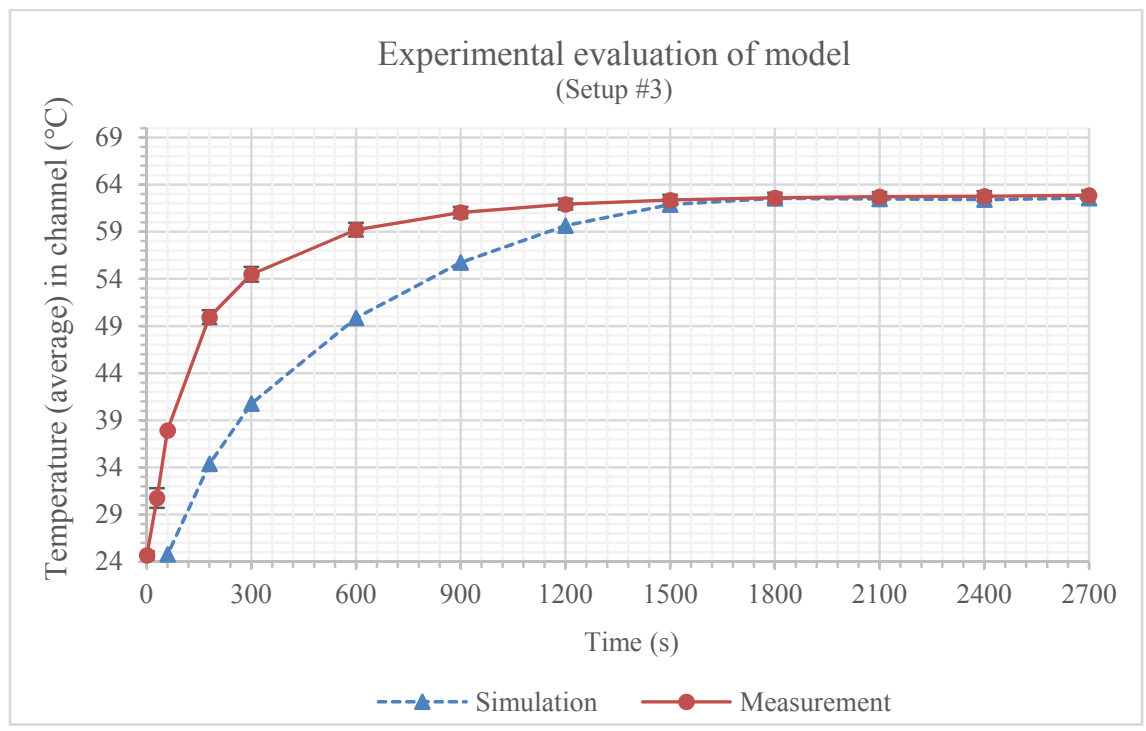

Figure 6: $\quad$ Model evaluation - Setup \#3.

\section{Conclusions}

A finite element model was proposed for the modelling of resistive heating elements for applications in microheating solutions for disposable molecular 
diagnostics, which was adaptable to multiple different commercially available heating elements. The model was presented and evaluated for experimental setups that imitated a disposable diagnostics platform with a replaceable microfluidic cartridge (chip) with typical dimensions and two different commercially available heating elements. Two setups were based on a self-regulating PTC ceramic heating element, and one on an etched foil heating element.

The temperature output of the experimental setup with the self-regulating element was estimated with an error of $0.31 \pm 0.17^{\circ} \mathrm{C}$ degrees around the set point when operated from a DC power supply, and with an error of $0.23 \pm 0.11^{\circ} \mathrm{C}$ when operated from batteries. The temperature output of the setup based on the etched foil heating element was estimated with an error of $1.12 \pm 0.62^{\circ} \mathrm{C}$ around the set point.

Furthermore, the experimental setups described in this paper were demonstrated to maintain target temperatures around set points in a predefined temperature range with better than $\pm 1^{\circ} \mathrm{C}$ precision.

The results discussed in this paper are preliminary and show an early stage of development. Nevertheless, proof was given that the proposed model was capable of estimating the heat output of microheating solutions in diagnostic devices, and as the in-house development of heating solutions proceeds, the precision of these estimations are bound to improve significantly.

\section{Acknowledgements}

The authors wish to thank for the support of the Sächsische AufbauBank provided under project SAB\#100144699, and the Estonian Research Council provided under research projects SF0140061s12, ETF8592 and IUT19-11, and the Foundation Archimedes through the Centre of Excellence CEBE (TK05U01) for supporting technology-oriented scientific projects in Estonia. Additionally, the authors would like to express their gratitude to Jüri Oleitšuk for the manufacturing of test structures.

\section{References}

[1] Wyzkiewicz, I., Grabowska, I., Chudy, M., Brzozka, Z., Jakubowska, M., Wisniewski, T. \& Dybko, A., Self-regulating heater for microfluidic reactors, Sens. Actuators B Chem., vol. 114, no. 2, pp. 893-896, Apr. 2006.

[2] Shioi, R., Umeya, K., Yonezuka, K. \& Senzaki, H., Heating element made of PTC ceramic material, Tdk Electronics Company Limited, 1981.

[3] Huybrechts, B., Ishizaki, K., \& Takata, M., The positive temperature coefficient of resistivity in barium titanate, J. Mater. Sci., vol. 30, no. 10, pp. 2463-2474, 1995.

[4] Meier, A. von., Electric power systems a conceptual introduction, Hoboken, N.J.: IEEE Press : Wiley-Interscience, 2006.

[5] Griffiths, D. J., Introduction to electrodynamics, Prentice hall, 1999.

[6] Kandlikar, S. G., Heat transfer and fluid flow in minichannels and microchannels, Elsevier, 2006. 
[7] Rood, P., A visual method of showing the high temperature coefficient of resistance of metals as compared with alloys, J. Opt. Soc. Am., vol. 16, no. 5, p. 357, Sep. 1928.

[8] Minco Products, Inc., Flexible heaters design guide HDG01121806(A), Minco Products Inc., 2007.

[9] Nagamine, K., Hase, T. \& Notomi, T., Accelerated reaction by loopmediated isothermal amplification using loop primers, Mol. Cell. Probes, vol. 16, no. 3, pp. 223-229, Jun. 2002.

[10] Bohlender, F. \& Buchlaub, N., PTC heating resistor, David \& Baader DBK Spezialfabrik elektrischer Apparate und Heizwiderstände GmbH, 1989. 